\title{
Atrial fibrillation with small subcortical infarcts
}

\author{
D K Jung, G Devuyst, P Maeder, J Bogousslavsky
}

\begin{abstract}
Objectives-To evaluate the characteristics of cardioembolic small (maximum lesion diameter $<1.5 \mathrm{~cm}$ ) subcortical infarcts (SSI) in patients with atrial fibrillation (AF).

Methods-Twenty seven patients with chronic AF and an isolated SSI established by CT/MRI in the anterior circulation (SSI-AF group) were evaluated and their characteristics compared with those of 45 age matched ( \pm 1 year) patients with SSI, but no arterial or cardiac embolic source (SSI-control group). Using the criterion of the presence or absence of established risk factors (hypertension or diabetes mellitus) for small artery disease (SAD), the SSI-AF group we also subdivided into two groups, SSI-AF-SAD+ $(n=22)$ and SSI-AF-SAD- $(n=5)$ and their characteristics compared.
\end{abstract}

Results-Although the lack of any significant difference in the distribution of hypertension and diabetes mellitus between the SSI-AF and SSI-control groups emphasises SAD as a common cause of infarct in SSI-AF, the presence of AFtogether with the higher frequency of neuropsychological disturbances in the SSI-AF group versus the SSI-control group $(15 \% v 2 \% ; p=0.066)$-favours cardioembolism as a potential cause of infarct in several patients. The characteristic factors seen more often in the SSI-AF-SAD- group compared with the SSI-AF-SAD+ group were secondary haemorrhagic transformation, faciobrachial pure motor weakness, subinsular involvement, and better recovery of neurological deficits.

Conclusions-The study suggests that either SAD or cardioembolism can be the cause of SSI in patients with AF. Atrial fibrillation is not always coincidental in patients with SSI and a clinical lacunar stroke. Certain clinical and radiological findings may be useful in differentiating cardioembolism from SAD in patients with SSI.

(F Neurol Neurosurg Psychiatry 2001;70:344-349)

Keywords: small subcortical infarcts; atrial fibrillation; cardioembolic infarcts

Small subcortical infarcts (SSI) are commonly considered to be synonymous with lacunar infarction caused by in situ small artery disease (SAD). However, although a large proportion of subcortical infarcts are accounted for by lacunar infarction, other forms may occur. ${ }^{12}$ Cardiac embolism, carotid disease, and other causes should not be overlooked. ${ }^{3-13}$ In lacunar infarction, a prevalence of up to $18 \%$ of cardioembolism has been reported, ${ }^{14}$ and several case reports provide radiological and pathological evidence for embolic lacunar infarction. ${ }^{15-18}$ By contrast, other studies suggest that embologenic cardiac disease is unlikely to be the cause of lacunar infarction. ${ }^{19-23}$ Using data from the Lausanne Stroke Registry for 27 patients with chronic atrial fibrillation (AF), one of the main cardiac sources of embolism, and an isolated, small (maximal lesion diameter $<1.5 \mathrm{~cm}$ ) subcortical infarct, established by CT/MRI, we systematically evaluated the epidemiology, risk factors for SAD, and clinicoradiological characteristics of SSI lesions probably attributable to a cardioembolic mechanism.

\section{Methods}

The SSI-AF group consisted of patients with an isolated small (maximal lesion diameter $<1.5 \mathrm{~cm}$ ) subcortical cerebral infarctproved by CT or MRI - in the territory of the internal carotid system and chronic AF. These patients were part of the Lausanne Stroke Registry and were admitted to our primary care centre between 1991 and 1997. In this study, all patients with first ever stroke were examined by a neurologist and the systematic investigations for each patient included brain CT (up to four examinations, the first within 7 days of the stroke) or MRI, with or without contrast, Doppler ultrasonography with spectral frequency analysis and B mode echotomography, 12 lead electrocardiography, blood tests (blood counts, liver, and renal function tests, venereal disease research laboratory test, total cholesterol, glucose, and sedimentation rate), and two dimensional echocardiography. Atrial fibrillation was diagnosed on the basis of either ECG results or 24-48 hour three lead ECG monitoring, together with history.

Hypertension was defined by history before stroke or high blood pressure (two or more blood pressure values $>160 / 95 \mathrm{~mm} \mathrm{Hg}$ ) during stay in hospital with the evidence of target organ damage (left ventricular hypertrophy, retinopathy, renal dysfunction) without previously recognised hypertension, diabetes mellitus (two or more fasting glucose concentrations $>6 \mathrm{mmol} / \mathrm{l}$ ), and hypercholesterolaemia (two or more fasting cholesterol concentrations $>6.5 \mathrm{mmol} / \mathrm{l})$. Concomitant factors, such as regular cigarette smoking, a history of migraine, or vascular claudication, were recorded according to the guidelines in our registry. Neck Doppler findings were grouped into four categories of normal, stenosis $<50 \%$, stenosis $>50 \%$, and occlusion. Coexisting 
Table 1 Age, sex, and factors for SAD in the SSI-AF and SSI-control groups

\begin{tabular}{lll}
\hline Group & SSI-AF & SSI-controls \\
\hline No & 27 & 45 \\
Sex (M/F) & $13 / 14$ & $21 / 24$ \\
Mean age (y) & 77 & 77 \\
& Range 56-87; SD 7 & Range 55-87; SD 7 \\
HTN & $21(78)$ & $39(87)$ \\
DM & $4(15)$ & $9(20)$ \\
Smoking & $5(19)$ & $10(22)$ \\
HC & $3(11)$ & $8(18)$ \\
Raised PCV & $4(15)$ & $6(13)$ \\
Claudication & $3(11)$ & $3(7)$
\end{tabular}

Values in parentheses are \%.

HTN=Hypertension; DM=diabetes mellitus; HC=hypercholesterolaemia; $\mathrm{PCV}=$ packed cell volume; $\mathrm{SAD}=$ small artery disease.

cardiac sources of embolism on two dimensional echocardiography included mitral or aortic valvular disease, prosthetic valves, akinetic left ventricular segment with or without thrombus, and global cardiac hypokinesia.

The radiologically identified SSIs were all relevant to the presenting clinical manifestations, including, but not limited to the four lacunar syndromes of pure motor hemiparesis, sensory motor stroke, ataxic hemiparesis, or pure sensory stroke. During a stay in hospital, all patients underwent several neuropsychological tests from a standard battery performed in our institution ${ }^{24}$ by experienced neurologists or neuropsychologists. Associated visuospatial dysfunction (hemineglect and hemianosognosia) and speech disturbance were recorded. Tests of hemineglect usually included observation of the patient's response to environmental stimuli, bilateral simultaneous sensory and visual stimulation, simple figure copying, drawing spontaneously, line bisection, picture scanning, and article reading. The number, frequency, side, duration, and time before stroke of transient ischaemic attacks, and the pattern of stroke onset were recorded according to the protocol of the Lausanne Stroke Registry. ${ }^{25}$ Functional status was measured on discharge using a five point scale, with 1 as no disability, 2 as mild disability (able to return to all activities, but with difficulty for certain activities), 3 as moderate disability (able to return with difficulty to only certain main activities), 4 as severe disability (not able to return to most activities), and 5 as death.

To determine whether the clinicoradiological features of SSI with AF could be distinguished from those of SSI due to SAD, an SSI-control group, consisting of age matched

Table 2 Echocardiographic results in the SSI-AF group

\begin{tabular}{lll}
\hline & $S S I-A F-A S D-$ & $S S I-A F-A S D+$ \\
\hline Myocardial abnormality & GH+LVT 1 & GH-LVT 4 \\
& GH-LVT 1 & FLVH-LVT 5 \\
Valvular abnormality & Pr MV 1 & Pr AV 1 \\
& - & FLVH and MR1 \\
Myocardial and valvular abnormality & - & 4 \\
LA Dilatation only & 2 & 5 \\
Normal or non-significant & & \\
\hline
\end{tabular}

$\mathrm{GH}=$ global hypokinesia; FLVH=focal left ventricular hypokinesia; LVT=left ventricular thrombosis; $M V=$ mitral valve; $M S=$ mitral stenosis; $M R=$ mitral regurgitation; $L A=l$ eft atrium; $\mathrm{AV}=$ aortic valve; $\mathrm{AS}=$ aortic stenosis; $\mathrm{Pr}=$ prosthetic; $+=$ with; $-=$ without.
( \pm 1 year) patients with SSI in the anterior circulation without cardiac embologenic source or significant internal corotid artery stenosis $(\geqslant 50 \%)$, was selected from patients with first stroke consecutively admitted to our hospital over the same period. The clinical characteristics and concomitant factors of the SSI-AF group were compared with those in the SSI-control group. To assess the potential role of AF itself as a probable cause in SSI, we compared the characteristics of patients with and without presumed SAD (presence of at least one of the two established risk factors for SAD - that is, hypertension or diabetes). The $\chi^{2}$ test and Fisher's exact test were used for statistical comparisons.

\section{Results}

The SSI-AF and SSI-control groups consisted of 27 and 45 patients, respectively; their mean ages and sex distributions are shown in table 1. The SSI-AF-SAD - and SSI-AF-SAD+ subgroups consisted of five (men/women 4/1) and 22 (men/women 9/13) patients with mean ages of 72 and 78 years, respectively.

VASCULAR CONCOMITANTS

Hypertension was the dominant factor for SAD in the SSI-AF (78\%) and SSI-control (87\%) groups, which contained four $(15 \%)$ and nine $(20 \%)$ patients with diabetes mellitus respectively (table 1 ). No statistical difference in the distribution of other risk factors (cigarette smoking, hypercholesterolaemia, raised packed cell volume, and limb claudication) was seen between the SSI-AF and SSI-control groups. The internal carotid artery Doppler ultrasound examinations showed that no patient had significant disease (stenosis $\geqslant 50 \%$ or occlusion) on both sides except one SSI-AF-SAD+ who had ipsilateral occlusion.

\section{COEXISTING CARDIAC SOURCES}

The echocardiographic findings are summarised in table 2. A cardiac pacemaker was implanted in one patient in the SSI-AF-SADgroup because of third degree atrioventricular block. Echocardiography was not performed on one SSI-AF-SAD + patient because of associated pulmonary disease. Valvular disorders were found in three $(17 \%)$ patients, two of whom had prosthetic mitral valve or rheumatic mitral sclerosis. Coexisting myocardial abnormalities were found in $12(44 \%)$ patients. Normal or non-significant aortic stenosis findings were found in seven (26\%) patients.

CLINICAL FINDINGS AND LOCATION OF INFARCTS These are shown in fig 1 . In the SSI-AF group, the lesion was identified by CT in 26 patients and by MRI in three patients; in $14(52 \%)$ patients, the lesion was on the right, whereas in $13(48 \%)$ it was on the left. In the SSI-control group, in $25(56 \%)$ patients, the lesion was on the right whereas in $20(44 \%)$ it was on the left. The distribution of lacunar syndromes did not differ significantly between the SSI-AF and SSI-control groups. Pure motor stroke (44\%), sensory motor stroke (38\%), and ataxic 


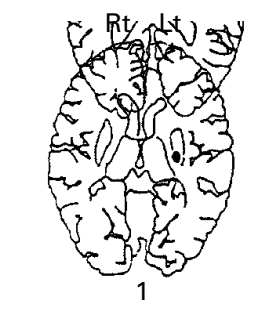

PM/anos, paraphasia
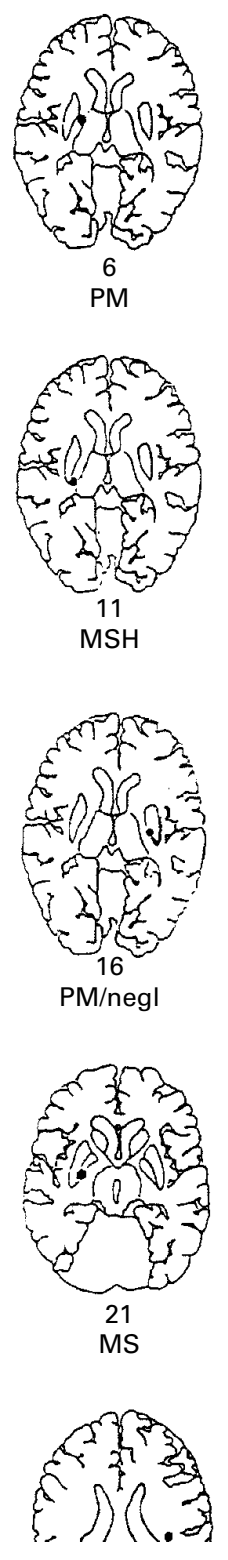

hemiparesis $(9 \%)$ were the main neurological syndromes. Sensory motor ataxia (4\%), sensory motor stroke with visual field defect $(2 \%)$, and ataxic hemiparesis with visual field defect $(2 \%)$ were uncommon. The lesion was located in the posterior internal capsule in 18 patients, the posterior internal capsule and lenticular nucleus in 11 , the periventricular white matter in five, the centrum ovale in four, the lenticular nucleus in three, the globus pallidus in two, the anterior internal capsule in one, and the thalamocapsular area in one. No significant difference was found in the distribution of lesion location between the SSI-AF and SSI-control groups. Four (15\%) patients in the SSI-AF group and one (2\%) in the SSI-control
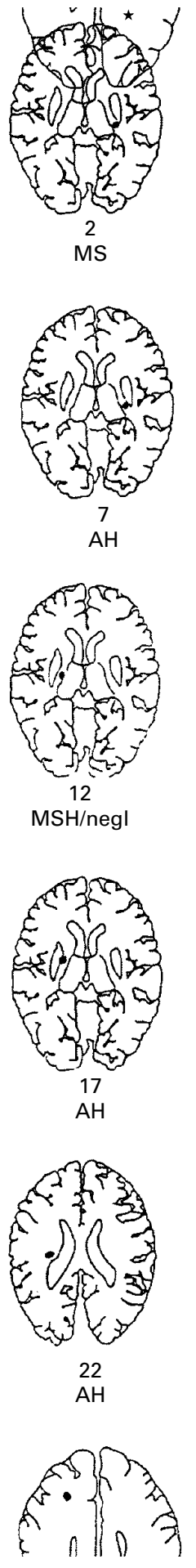

Figure 1 Infarct locations and associated clinical findings in the SSI-AF group. Infarct locations in five (1-5) SSI-AF-ASD-patients and in 22 (6-27) SSI-AF-ASD+ patients. $P M=$ Pure motor stroke; $M S=$ motor/sensory stroke; $M S H=$ motor/sensory stroke with visual field defect; $A H=$ ataxic hemiparesis; FB type=faciobrachial type of motor weakness; anos=anosognosia; negl=hemineglect; ${ }^{*}$ haemorrhagic transformation. 


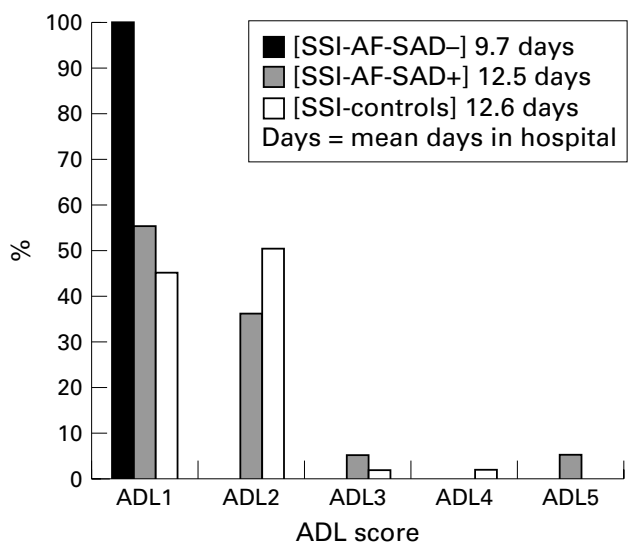

Figure 2 Activities of daily living $(A D L)$ scores on discharge in SSI-AF-SAD-, SSI-AF-SAD+, and SSI-controls. $D=$ mean days of stay in hospital.

group had dysphasia or visuospatial dysfunction $(p=0.066)$. All neuropsychological dysfunctions in the SSI-AF group subsided within 3 days. One patient in the SSI-control group had anosognosia, the lesion being in the right periventricular white matter.

PREVIOUS TRANSIENT ISCHAEMIC ATTACKS AND STROKE ONSET

Two (9\%) patients in the SSI-AF-SAD+ group and five $(11 \%)$ in the SSI-control group reported one episode of transient ischaemic attack ipsilateral to their subsequent infarct. Stroke was immediately stabilised in five patients in the SSI-AF-SAD- group, 20 (91\%) in the SSI-AF-SAD+ group, and $35(80 \%)$ in the SSI-control group, fluctuated in four $(18 \%)$ patients in the SSI-AF-SAD+ group and five $(11 \%)$ in the SSI-control group, and progressed gradually in four $(18 \%)$ patients in the SSI-AF-SAD+ group and four $(9 \%)$ in the SSI-control group. One patient died due to cardiac failure 36 days after stroke in the SSIAF-ASD- group. No statistical significance was found in the prevalence of transient ischaemic attacks and fluctuation or progression of neurological deficits between the SSI-AF and SSI-control groups or between the SSI-AF-ASD- and SSI-AF-ASD+ groups.

TREATMENT AND SHORT TERM EVOLUTION One $(20 \%)$ patient in the SSI-AF-ASD- group and $12(55 \%)$ in the SSI-AF-SAD+ group were treated with aspirin, four $(80 \%)$ patients in the SSI-AF-ASD- group and eight $(36 \%)$ in the SSI-AF-ASD+ group were anticoagulated, and the remaining two patients in the SSI-AF$\mathrm{ASD}+$ group did not receive antithrombotic drugs because of a bleeding tendency (thrombocytopenia or liver disease). All patients except one with gastric intolerance in the SSIcontrol group were treated with aspirin. Figure 2 shows the functional disability on discharge and the mean duration of stay in hospital. All five patients in the SSI-AF-ASD- group and 12 $(55 \%)$ in the SSI-AF-ASD+ group had no disability on discharge. The mean duration of stay in hospital in the SSI-AF-ASD- group was shorter than in the SSI-AF-ASD+ group (9.7 days $v 12.5$ days). Functional disability at the time of discharge after adjustment for the duration of stay in hospital was not significantly different between the SSI-AF and SSI-control groups.

\section{Discussion}

Cardioembolic subcortical infarcts have been diagnosed mainly from the presence of cardioembolic sources, usually without considering the clinical characteristics. This can be explained by the fact that only a few studies documented clinical features of such infarcts. As far as we know, this is the first attempt to systematically evaluate the characteristics of potentially cardioembolic subcortical infarcts in patients with atrial fibrillation.

Whereas hypertension and diabetes mellitus are not specific risk factors for small artery disease, our findings that the frequency of risk factors for small artery disease did not differ significantly between the SSI-AF and SSIcontrol groups emphasise small artery disease as a cause of subcortical infarcts with atrial fibrillation. Some previous studies have demonstrated hypertension and diabetes mellitus to be potent risk factors for subcortical infarcts. ${ }^{19} 2627$ However, atrial fibrillation as a potential mechanism of subcortical infarct formation should not be overlooked. Now that hypertension is better controlled because of more effective treatments, and is less likely to be the sole cause of subcortical infarcts, reports increasingly suggest an embolic mechanism as a cause of the infarcts. ${ }^{15-18}{ }^{28}$ In a recent clinical study that strongly supported cardiac embolic sources as a cause of subcortical infarcts, ${ }^{29}$ five (17\%) of 29 new infarcts associated with cardiac surgery were subcortical. Recently, on the basis of pathological studies, Lammie et a ${ }^{30}$ suggested that embolic occlusion of the small penetrating vessels may be more common than generally assumed in elderly hypertensive patients.

Subcortical infarcts can lead to various neuropsychological disturbances. ${ }^{31-33}$ Our data do not allow us to draw a conclusion as to whether the mechanism of neuropsychological disturbances in cardioembolic subcortical infarcts is similar to that of subcortical infarcts due to small artery disease. The few cases and inherent selection biases must be taken into consideration.

Acute onset, transient duration, and lateralisation of neuropsychological dysfunction suggest that stroke is the main cause, and an insufficient cardiac output for the maintenance of an adequate brain perfusion, resulting from chronic atrial fibrillation and associated myocardial dysfunction, could be an additional factor. ${ }^{34}{ }^{35}$ Another possible explanation for the higher incidence of neuropsychological disturbances in the SSI-AF group is that some cardiac emboli obstructed the deep perforating branch, whereas others lodged in the pial branches, causing cortical neuropsychological disturbances. ${ }^{15}$ 36-38 Transient disturbance of the cortical or subcortical blood flow resulting from fragmentation and migration of an embolus could be another possibility. ${ }^{39}$ The cortical ischaemic lesion may have been invisible due to 
early recanalisation of emboli, the small size of the emboli, or limitations in the ability of brain imaging to demonstrate an associated cortical lesion.

The fluctuation and progression of neurological deficits in the SSI-AF group does not seem helpful in predicting the mechanism of subcortical infarct formation. Although stepwise or gradual onset due to small artery disease has been reported, ${ }^{40}{ }^{41}$ embolic infarcts can also have various modes of onset, and several factors combine to cause progression of neurological deficits in patients with subcortical infarcts. ${ }^{42-44}$

In two patients in the SSI-AF-ASD- group, the lesions were in the posterior internal capsule, which agrees with the previous published location in embolic SSI. ${ }^{15} 1645$ One of these two patients showed secondary haemorrhagic transformation, which suggests an embolic mechanism, whereas the other patient had anosognosia and paraphasia.

In one patient in the SSI-AF-ASD- group, the lesion was in the subinsular area (the extreme capsule), and, considering the transient faciobrachial motor weakness seen in this case, we cannot rule out the possibility of ischaemic damage at other sites, including the cerebral cortex. The lesion site suggests an embolic mechanism, as the extreme capsule is supplied by the adjacent cortical branch.

Recovery from neurological deficits in the SSI-AF-ASD - group occurred earlier and was better than in the SSI-AF-ASD+ group; however, these differences did not reach significance, partly due to the few patients. Less severe ischaemia due to early recanalisation of an occluded single perforating artery in the SSI-AF-ASD- group may be an explanation for this excellent prognosis.

Small artey disease cannot be definitely excluded as a possible cause of stroke in the SSI-AF-ASD- group. ${ }^{46}{ }^{47}$ However, secondary haemorrhagic transformation, faciobrachial motor weakness with extreme capsule involvement, the remarkably favourable short term prognosis, and atrial fibrillation as a strong embolic risk factor strongly suggest an embolic mechanism in the SSI-AF-ASD- group. The increase in prevalence of atrial fibrillation with age ${ }^{48}$ may account for the older mean age (72 years) of patients in the SSI-AF-SADgroup.

In conclusion, either cardioembolism or small artery disease can be a causative factor in patients with subcortical infarcts with atrial fibrillation, and atrial fibrillation is not always coincidental in patients with subcortical infarcts and a clinical lacunar stroke. Certain clinical and radiological features may be useful in distinguishing cardioembolic from subcortical infarcts due to small artery disease.

1 Donnan GA, Norrving B, Bamford JM, et al. Subcortical infarction: classification and terminology. Cerebrovasc Dis 1993;3:248-51.

2 Bogousslavsky J. The plurality of subcortical infarction [editorial]. Stroke 1992;23:629-31.

3 Levin RL, Lagreze HL, Dobkin JA, et al. Large subcortical hemispheric infarction: presentation and prognosis. Arch Neurol 1988;45:1074-7.
4 Donnan GA, Bladin PF, Berkovic SF, et al. The stroke syndrome of , Bladin PF, Berkovic SF, et al. The stroke syn70 .

5 Ringelstein EB, Biniek R, Weiller C, et al. Type and extent of hemispheric brain infarcts and clinical outcome in early and delayed middle cerebral artery recanalisation. Neurology 1992;42:289-98

6 Hupperts RMM, Lodder J, Heuts-van Raak EPM, et al. Infarcts in the anterior choroidal artery territory: anatomical distribution, clinical syndromes, presumed pathogenesis and early outcome. Brain 1994;117:825-34.

7 Aleksic SN, George AE. Pure motor hemiplegia with occlusion of the extracranial carotid artery. $\mathcal{F}$ Neurol Sci 1973;19: $331-9$.

8 Barinagarrementeria F, Del Brutto OH. Lacunar syndrome due to neurocysticercosis. Arch Neurol 1989;46:415-17.

9 Millikan $\mathrm{CH}$, Futrell N. The fallacy of the lacunar hypothesis. Stroke 1990;21:1251-7.

10 Waterston JA, Brown MM, Butler P, et al. Small deep cerebral infarcts associated with occlusive internal carotid artery disease: a hemodynamic phenomenon? Arch Neurol artery disease: a

11 Bogousslavsky J, Regli F, Maeder P. Internal large-artery and lacunar infarction. Cerebrovasc Dis 1991;1:154-9.

12 Park YD, Belman AL, Kim TS, et al. Stroke in pediatric acquired immunodeficiency syndrome. Ann Neurol 1990; 28:303-11.

13 Fredericks PK, Leflowits DS, Challa VR, et al. Cerebral vasculitis associated with cocaine abuse. Stroke 1991;22:1437-9.

14 Horowitz DR, Tuhrim S, Weinberger JM, et al. Mechanisms in lacunar infarction. Stroke 1992;23:325-7.

15 Futrell N, Millikan C, Watson BD, et al. Emboli stroke from a carotid arterial source in the rat. Neurology 1989;39: 1050-6.

16 Cacciatore A, Russo LS Jr. Lacunar infarction as an embolic complication of cardiac and arch angiography. Stroke 1991; 22:1603-5.

17 Laloux P, Brucher JM. Lacunar infarctions due to cholesterol emboli. Stroke 1991;22:1440-4.

18 MacDonald RL, Kowalczuk A, Johns L. Emboli enter penetrating arteries of monkey brain in relation to their size. Stroke 1995;26:1247-51

19 Gandolfo C, Caponnetto C, Del Sette M, et al. Risk factors in lacunar syndromes: a case-control study. Acta Neurol Scand 1988;77:22-6.

20 Foulkes MA, Wolf PA, Price TR, et al. The stroke data bank: design, methods, and baseline characteristics. Stroke 1988; 19:547-54.

21 Van Merwijk G, Lodder J, Bamford J, et al. How often is non-valvular atrial fibrillation the cause of brain infarction? f Neurol 1990;237:205-7.

22 Norrving B, Staff G. Pure motor stroke from presumed lacunar infarct. Incidence, risk factors and initial course. Cerebrovasc Dis 1991;1:203-9.

23 Mast H, Thompson JLP, Voller H, et al. Cardiac sources of embolism in patients with pial artery infarcts and lacunar lesions. Stroke 1994;4:420-5.

24 Assal G. Batterie des examens neuro-psychologiques du CHUV. Lausanne, Switzerland: Centre Hospitalier Universitaire Vaudois, 1985.

25 Bogousslavsky J, Van Melle G, Regli F. Lausanne Stroke Registry Group. The Lausanne Stroke Registry: analysis of 1000 consecutive patients with first stroke. Stroke 1988;19: 1083-92.

26 Bamford J, Sandercock P, Jones L, et al. The natural history of lacunar infarction: Oxfordshire Community Stroke Project. Stroke 1987;18:545-51.

27 Arboix A, Marti-Vilalta JL, Garcia JH. Clinical study of 227 patients with lacunar infarcts. Stroke 1990;21:842-7.

28 Fisher CM. Capsular infarcts: the underlying vascular Fisher CM. Capsular infarcts: the
lesions. Arch Neurol 1979;36:65-73.

29 Libman RB, Wirkowski E, Neystat M, et al. Stroke Libman RB, Wirkowski E, Neystat $\mathrm{M}$, et al. Stroke
associated with cardiac surgery: determinants, timing, and stroke subtypes. Arch Neurol 1997;54:83-7.

30 Lammie GA, Brannan J, Wardlaw JM. Incomplete lacunar infarction (type Ib lacunes). Acta Neuropathol 1998;96:163-71.

31 Fisher CM. Lacunar infarcts-a review. Cerebrovasc Dis 1991; $1: 311-20$

32 Kobayashi S, Okada K, Yamashita K. Incidence of silent lacunar lesion in normal adults and its relation to $\mathrm{CBF}$ and risk factors. Neurology 1991;22:1379-83.

33 Tatemichi TK, Desmond I, Prohovnik I, et al. Frontal lobe syndrome with memory loss after capsular genu infarct [abstract] Cerebrovasc Dis 1992;2:195.

34 Murgatroyd FD, Camm AJ. Atrial arrhythmia. Lancet 1993; 341:1317-22.

35 Ott M, Breteler MMB, Bruyne MC, et al. Atrial fibrillation and dementia in a population-based study. The Rotterdam study. Stroke 1997;28:316-22.

36 Ringelstein EB, Koseborke S, Holling A, et al. Computed tomographic patterns of proven embolic brain infarctions. Ann Neurol 1989;26:759-65.

37 Tuszynski MH, Petito CK. Risk factors and clinical manifestations of pathologically verified lacunar infarctions. Stroke 1989;20:990-9.

38 Ay H, Oliveira-Filho J, Buonanno FS, et al. Diffusionweighted imaging identifies a subset of lacunar infarction associated with embolic source. Stroke 1999:30:2644-50.

39 Sacco RL, Toni D, Mohr JP. Classification of ischemic stroke. In: Barnet HJM, Mohr JP, Stein BM, et al, eds. Stroke: pathophysiology, diagnosis, and management. New Stroke: pathophysiology, diagnosis, and mana

40 Mohr JP. Lacunes. Stroke 1982;13:3-13.

41 Gautier JC. Stroke in progression. Stroke 1985;16:729-33. 
42 Fisher CM, Pearlman A. The non-sudden onset of cerebral embolism. Neurology 1967;17:1025-32.

43 Mohr JP, Caplan LR, Melski JW, et al. The Harvard Cooperative Stroke Registry: a prospective registry. Neurology 1978;28:754-62.

44 Nakamura K, Saku Y, Ibayashi S, et al. Progressive motor deficits in lacunar infarction. Neurology 1999;52:29-33.

45 Arboix A, Varti-Vilata JL. Presumed cardioembolic lacunar infarcts [letter]. Stroke 1992;23:1841-2.
46 Bamford J, Sandercock P, Jones L, et al. The natural history of lacunar infarction. The Oxfordshire Community Stroke . Stroke 1987:18:545-1.

47 Sacco SE, Whisnant JP, Broderick JP, et al. Epidemiological characteristics of lacunar infarcts in a population. Stroke 1991;22:1236-41.

48 Feinberg WM, Blackshear JL, Laupacis A, et al. Prevalence, age distribution and gender of patients with atrial fibrillation. Arch Intern Med 1995;155:469-73.

\section{HISTORICAL NOTE}

\section{Henry Hun (1854-1924)}

Neurology in upper New York State was pioneered by the Hun family. ${ }^{1}$ Thomas Hun MD (1808-unknown), the neurological patriarch, completed medical studies at the University of Pennsylvania and spent 6 years of postgraduate study in Europe. ${ }^{1}$ On his return, he helped found the Albany Medical College (1839), where he was appointed Professor of the Institutes of Medicine. Long before neurology was a recognised specialty in the United States, he gave lectures on the nervous system, held weekly neurological clinics with medical students, and stimulated his sons Edward and Henry to pursue neurological careers.

Edward Reynolds Hun MD (1842-1880), the eldest son, graduated from the College of Physicians and Surgeons in 1866 and practiced in Albany. ${ }^{2} \mathrm{He}$ was appointed pathologist to the New York State Lunatic Asylum at Utica, and in 1876 accepted the Chair of Diseases of the Nervous System at Albany Medical College. Edward was one of the 35 original members of the American Neurological Association, founded in 1875. Unfortunately, he died prematurely.

Henry Hun MD (1854-1924) graduated from Harvard Medical School in 1879 and spent more than 2 years of postgraduate study, encompassing Vienna, Heidelberg, Berlin, Paris, and London. ${ }^{2}$ His interest in neurology was further stimulated by exposure to Jean-Martin Charcot (1825-93) at the
Salpêtrière Hospital. On return, he was appointed Professor of Diseases of the Nervous System in the Albany Medical College (1884-1914). He wrote several treatises, including $A$ guide to American medical students in Europe (1883), Syllabus of a course of lectures on the diseases of the nervous system (1901/ 1902; 1907), and An atlas of the differential diagnosis of the nervous system (1913; 1914; 1922). He also served as President of the Association of American Physicians (1910), and President of the American Neurological Association (1914).

Henry Hun is now most often recognised for his detailed clinicopathological description of the lateral medullary syndrome. Hun's 1897 report included a very detailed (albeit delayed) pathological report from the neuropathologist Ira Van Gieson (1866-1913) after the patient's death in $1893 .{ }^{3}$ Hun's 53 year old patient had acutely developed ipsilateral ptosis and anhidrosis (but reportedly not miosis), diplopia, ipsilateral analgesia and thermanaesthesia of the face, dysphagia, dysphonia, dysarthria, ipsilateral appendicular ataxia, postural instability with a tendency to fall toward the side of the lesion, gait ataxia, contralateral analgesia and thermanaesthesia of the body, and vomiting. Pathological findings included extensive posterior circulation atherosclerosis, affecting especially the basilar artery, and infarction of the rostral lateral medulla, encompassing the descending trigeminal nucleus and tract, the nucleus ambiguous, the lateral spinothalamic tract, the ventral spinocerebellar tract, fasciculi of the glossopharyngeal and vagal nerves, and the inferior cerebellar peduncle. Hun concluded that the manifestations resulted from left posterior inferior cerebellar artery occlusion. Hun's report was partially preceded by Adolf Wallenberg's detailed clinical (but not pathological) description in $1895,{ }^{45}$ and both of these reports were partially anticipated by others. ${ }^{45}$ Based on his knowledge of neuroanatomy, Wallenberg had correctly localised his patient's lesion to the lateral medulla and attributed it to occlusion of the ipsilateral posterior inferior cerebellar artery, ${ }^{4}$ but it was not until 1901,4 years after Hun's clinicopathological report, that Wallenberg reported the pathological findings on his patient, who died in 1899 .

VA Medical Center, Great Lakes VA

D J LANSKA

Healthcare System, 500 E Veterans Street,

Tomah, Wisconsin 54660, USA

Douglas.Lanska@med.va.gov

1 Lawyer T Jr. Development of neurology in upper New York State: Albany neurology. In: Denny-Brown D, et al, eds. Centennial anniveration: 1875-1975. New York: Springer, 1975: ation: $1875-1$

2 Tilney F, Jelliffe SE, eds. Semi-centennial anniversary volume of the American Neurological Association: 1875-1924. American Neurological Association, 1924:111-13; 189-90.

3 Hun $H$. Analgesia, thermic anaesthesia, and ataxia, resulting from foci of softening in the medulla oblongata and cerebellum, due to occlusion of the left inferior posterior cerebellar artery. A study of the course of the sensory and co-ordinating tracts in the medulla oblonand co-ordinating tracts in the m

4 Wallenberg A. Acute bulbäraffection (embolic der art. cerebellar. post. inf. sinstr?). Archiv für der art. cerebellar. post. inf. sinstr?). Archiv für
Psychiatrie und Nervenkrankheiten 1895;27: Pos 40.

504-40

Wolf JK. Wallenberg's syndrome. In: The classical brain stem syndromes: translations of the original papers with notes on the evolution of clinical neuroanatomy. Springfield, Illinois: Charles C Thomas, 1971:113-36.

6 Wallenberg A. Anatomischer Befund in einem als "acute Bulbär-affection (Embolie der Art. cerebellar. post. inf. sinistr?)" bescreidenen Falle. Archiv für Psychiatrie und Nervenkrankheiten 1901;34:923-59. 